Recibido: 24 enero 2014

Aprobado: 21 marzo 2014

\section{BARRANQUILLA Y LA MODERNIZACIÓN DEL DELTA DEL RÍO MAGDALENA (1842-1935)*}

Carlos Bell Lemus**

Universidad del Atlántico, Colombia

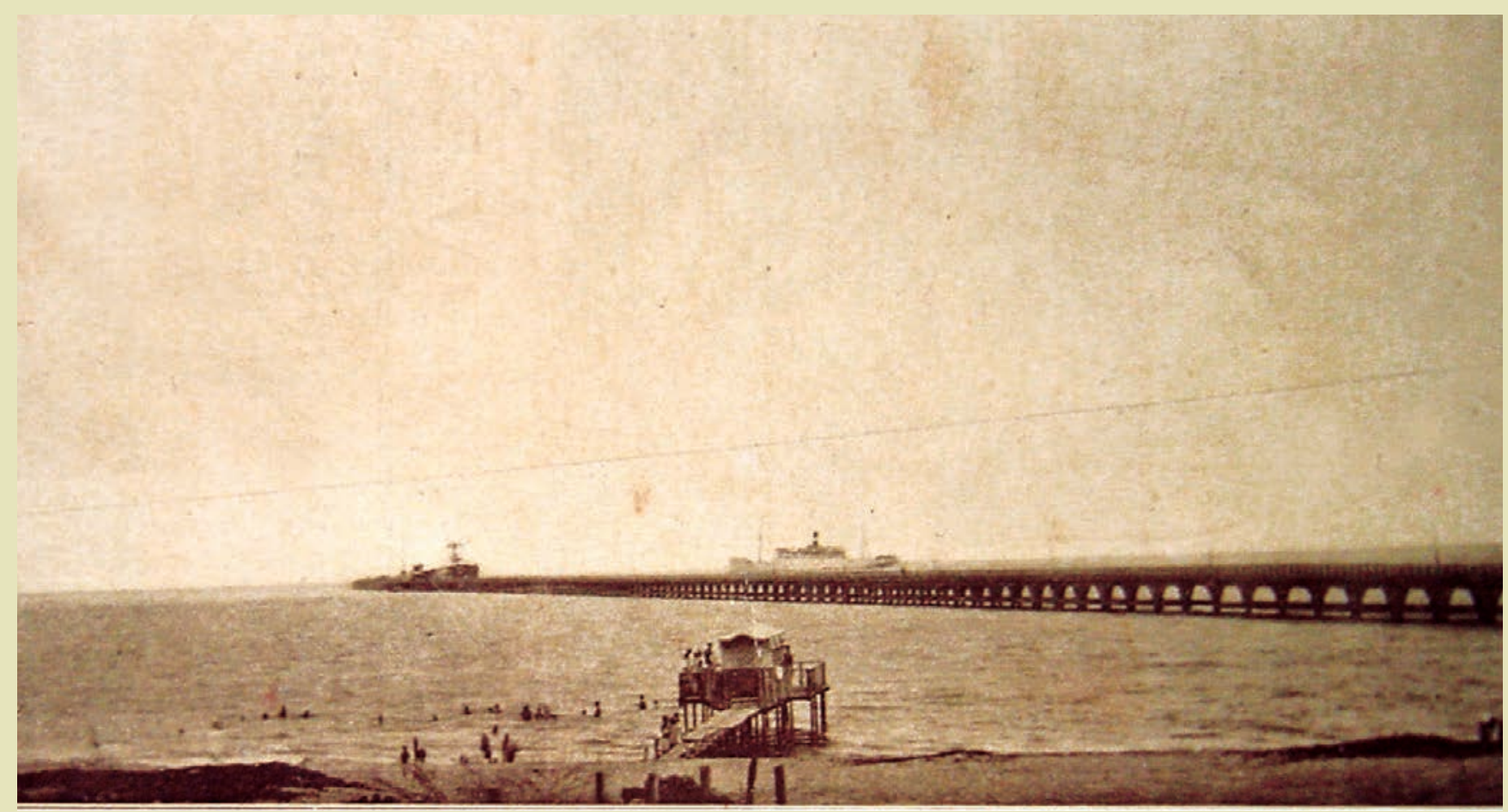

* Este artículo es derivado de la tesis doctoral Barranquilla procesos de modernización y movimiento moderno en arquitectura, del programa de Doctorado en Arte y Arquitectura de la Universidad Nacional de Colombia.

** Arquitecto especialista en Planeación y Gestión del Desarrollo Urbano y Regional, magíster en Proyectos de Desarrollo Social, actualmente es candidato a doctor en Arte y Arquitectura por la Universidad Nacional de Colombia. Correo electrónico: carlosbell@mail. uniatlantico.edu.co

\begin{abstract}
RESUMEN
Este ensayo hace parte de un capítulo de la tesis doctoral -de carácter histórico- en desarrollo, titulada Barranquilla procesos de modernización y movimiento moderno en arquitectura, del programa de Doctorado en Arte y Arquitectura de la Universidad Nacional de Colombia, y describe cómo el delta del Río Magdalena fue transformado físicamente, desde mediados del siglo XIX, para convertir su maleable geografía en un territorio que facilitara los procesos de modernización que requería Colombia en ese entonces, para mejorar la movilidad, incrementar el volumen de carga y potenciar el comercio internacional. A su paso, la construcción del ferrocarril, los tajamares de Bocas de Ceniza y la llegada de la aviación permitieron a Barranquilla su inserción a las dinámicas económicas e industriales nacionales, y la posicionaron desde 1842 hasta 1935 como el principal puerto multimodal sobre el Caribe colombiano y en la principal puerta de entrada a la modernidad del país.
\end{abstract}

\section{PALABRAS CLAVE}

Delta del río Magdalena, proyectos de movilidad, modernización, construcción del territorio. 


\section{BARRANQUILLA AND MODERNIZATION OF THE MAGDALENA RIVER DELTA (1842-1935)}

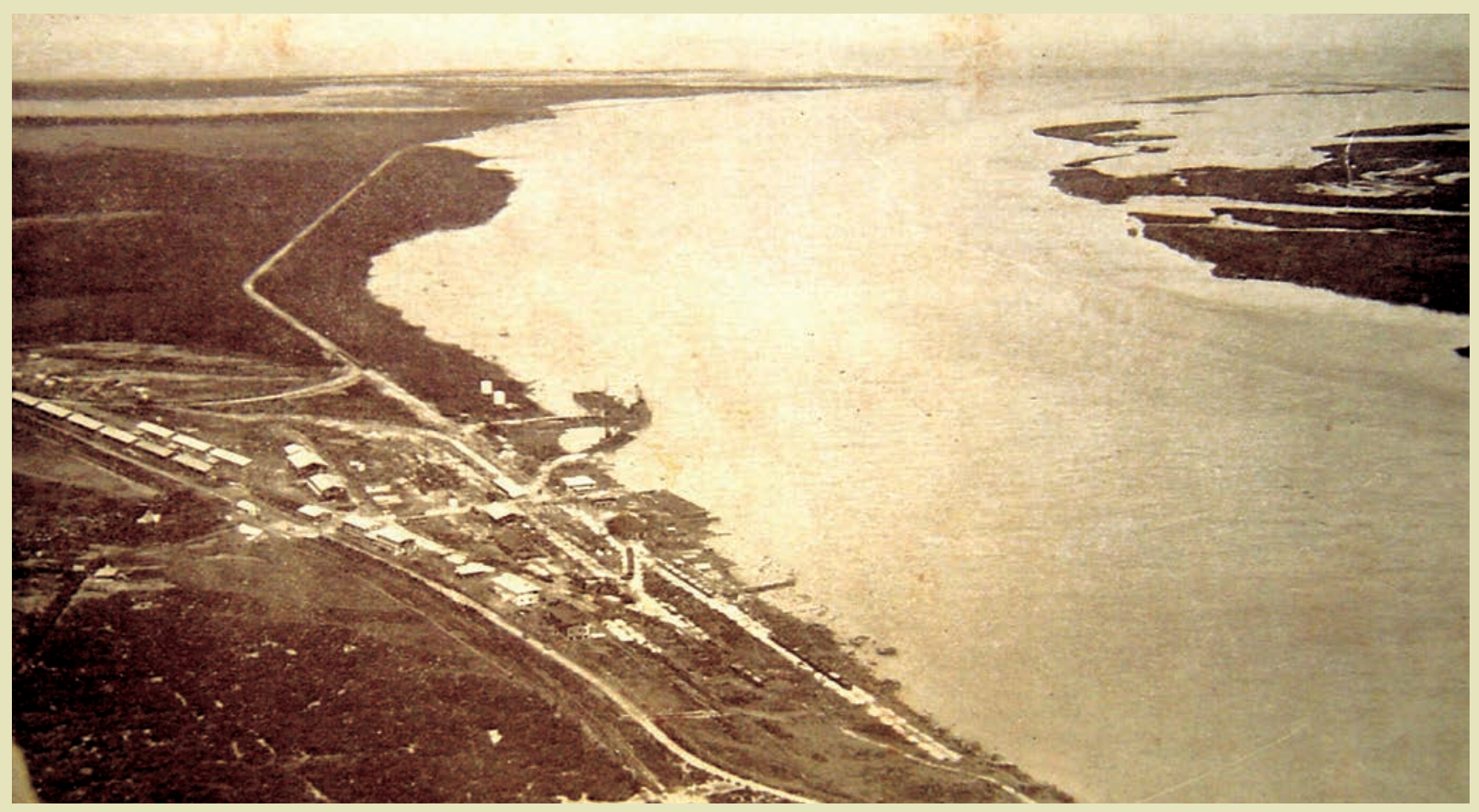

\section{ABSTRACT}

The territory of the delta of the Magdalena River from mid-nineteenth century was subjected to a modernization process which improved mobility, freight and international trade in Colombia. In the case of Barranquilla, railway construction, the breakwaters of Bocas de Ceniza and the advent of aviation led to the insertion and use of this space into economic and industrial dynamics that would position in this period (1842-1935) to the multimodal city as the main port on the Colombian Caribbean, and the gateway to modernity to the whole country

\section{KEY WORDS}

Delta of the Magdalena river; Projects of mobility; modernization; construction of the territory.

Campamento de "Las Flores" sobre el río Magdalena, 1928.

Fuente: Directorio Comercial Pro-Barranquilla. 1928 


\section{INTRODUCCIÓN}

Barranquilla es una ciudad ubicada a $7 \mathrm{~km}$. de la desembocadura del río Magdalena sobre el mar Caribe al norte de Colombia, cuya estratégica posición geográfica le permitió a través de las dinámicas de modernización propias del capitalismo industrial del siglo XIX y principios del $X X$, convertirse en el principal puerto de Colombia hasta mediados de los años treinta y para lo cual se vio abocada a realizar una serie de transformaciones físicas al delta del río. De esa manera, fue posible que la ciudad se integrara a los circuitos mundiales del comercio internacional y se convirtiera así, durante ese periodo, en la puerta de entrada de la modernidad en Colombia.

Tres acciones territoriales fueron fundamentales para lograr ese cometido, todas relacionadas con la mecanización de la movilidad. Primero la construcción del ferrocarril, que acercaría los primitivos lugares de desembarco en la bahía de Sabanilla y Puerto Colombia a las barrancas sobre los caños donde surgió Barranquilla; luego los tajamares de Bocas de Ceniza que permitirían la llegada de buques de mayor calado a la terminal fluvial de la ciudad y, por último, el desarrollo de la aviación con la construcción del hidropuerto de Veranillo que como un atajo al aislamiento del delta, facilitaría el uso de las aeronaves para superar la barrera física de la accidentada geografía colombiana, permitiendo fortalecer los vínculos comerciales entre el interior del país y la Costa Caribe colombiana.

De modo que el tren, el barco y el avión gestaron la transformación del delta del río Magdalena y convertiría a Barranquilla en un puerto multimodal por excelencia a principios del siglo $X X$ como se verá a continuación.

\section{El ferrocarril y la construcción del territorio de Barranquilla}

Debemos regocijarnos al ver establecida, fuera de toda duda, la libertad de esta porción interesante del nuevo mundo [...] se ha abierto un campo nuevo, rico, vasto y accesible... Accesible tanto para nosotros, como para nuestras colonias. Además de esto Inglaterra es la nación que Colombia imitará y favorecerá (Aprile, 1992, p. 16).

Una vez que consolidó su independencia, Colombia empezó a mirar otros referentes para desarrollar su nueva condición de Estado-nación independiente. Para ello recurrió, como muchos países latinoamericanos, a seguir el ejemplo del nuevo imperio de Gran Bretaña, que, soportado en sus avances en tecnología, industria, comercio mundial e innovaciones en los medios de transporte, se había tornado en el ejemplo de modernización industrial para copiar, por las recientes repúblicas independientes de Hispanoamérica.

De manera que, para marchar al ritmo de esa nueva concepción del mundo, las repúblicas liberales recién conformadas se vieron obligadas a introducir rápidos cambios en los paisajes naturales y en la morfología de las ciudades. Según José Luis Romero, desde 1880 muchas ciudades latinoamericanas comenzaron a experimentar nuevos cambios, tanto en su estructura social como en su fisonomía, a tal punto que los viajeros europeos se sorprendían de esas transformaciones que hacían irreconocible una ciudad en tan solo veinte años (Romero, 1999).

Estos cambios fueron más notorios en las ciudades capitales, y en puertos como Barranquilla por donde entraban mercancías y salían materias primas, y habitaba una comunidad signi- 


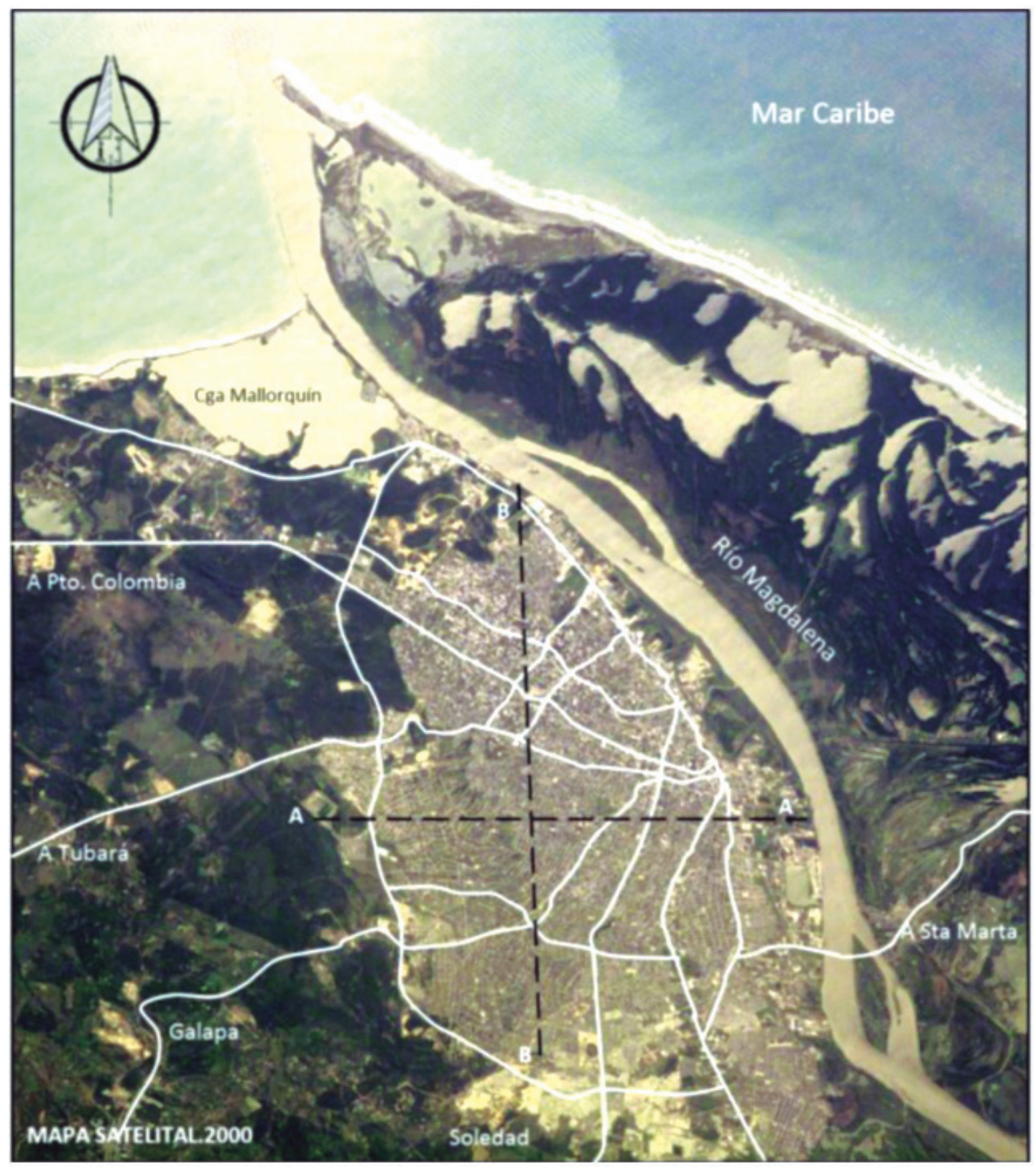

ficativa de comerciantes extranjeros. Estas nuevas burguesías, con una mentalidad abierta al mundo, hallaron en esas circunstancias el escenario propicio para importar los gustos, Fuente: elaboración del autor. el arte, la arquitectura, el espacio urbano cosmopolita. Allí se recibía primero que en otras ciudades del país, el correo de París, Londres o Nueva York (Giraldo \& Viviescas, 1995).

Los protagonistas principales de estos cambios culturales, económicos y urbanísticos en América Latina fueron un puñado de aventureros y empresarios extranjeros que, motivados por la gran movilidad social que permitían los negocios y su rápida capitalización, se arriesgaron a migrar hacia los diversos países latinoamericanos, para promover empresas, hacer alianzas con los grupos nacionales, crear bancos, casas comerciales e industrias; e imprimirles a estos asentamientos una espacialidad y un ritmo, al tiempo, similar al que transcurría en el mundo industrializado europeo. Asumieron el papel de liderazgo en la construcción de un mundo fundamentado en una vivencia del presente: "en la búsqueda 
de la realidad real. No en la búsqueda del Edén terrestre ni en la eternidad sin fechas", como lo expresa Octavio Paz (Giraldo \& Viviescas, 1995, p. II6).

Para la construcción de ese mundo real, que le da la conexión con el presente vital, la sociedad occidental del s. XIX obtuvo su gran apoyo logístico en el uso práctico de las ciencias físicas y naturales, para materializar su concepción del mundo y organizar los pueblos, diseñar sus hábitats, industrializar los procesos de producción, mecanizar el transporte, transformar los ecosistemas naturales en geografía, y apropiarse de los territorios. Con esas premisas subyacentes propias de la modernización, con la cual intervenir cualquier lugar del planeta, Barranquilla -un caserío irrelevante para los intereses de la Corona española en el s. XVIII- transformaría entonces su naturaleza en territorio, abocada por el imperativo de vincular a Colombia con el comercio internacional.

A mediados del s. XIX, Cartagena, que había sido el principal puerto durante la Colonia, había perdido su dinamismo comercial, pues el canal del Dique -vía de comunicación fluvial entre Cartagena y el río Magdalena-, se colmataba con frecuencia en varios tramos impidiendo la navegación; además, la otra posibilidad que existía para conectar a Colombia con el comercio internacional, que era por la desembocadura del río Magdalena, resultaba complicada, pues las Bocas de Ceniza eran inestables y llenas de barras de arena. Por ello, en Sabanilla, una de las bahías que se formaban en el delta del río -cerca de Barranquillacon una profundidad que permitía fondear a un kilómetro de la costa buques de 1000 a I 500 toneladas, se habilitó como puerto de fondeo en I820. En una compleja operación de transbordo de mercancías y pasajeros, a bongos y barcos de menor calado, se iniciaba la travesía por el canal de la Piña, entre las zonas de manglares hasta llegar a Barranquilla. O se iba a "pie, a caballo o en carretas arrastradas por bestias por un camino de $25 \mathrm{~km} \mathrm{y}$ gastaba un día de luz" (Poveda, 2002, p. 62).

A raíz de estos inconvenientes que hacían onerosa e ineficiente la actividad portuaria, en I 865 el Estado Soberano de Bolívar tomó la iniciativa de construir un ferrocarril entre Sabanilla y Barranquilla. Como carecía de recursos fiscales, acudió a otorgar "un privilegio del Estado a un particular", a dos inversionistas locales: Ramón Santodomingo Vila y Ramón Jimeno Collante, quienes, después de muchos disputas y atrasos, deciden subcontratar con la casa comercial alemana Hoenigsberg, Wessels y Cía. la construcción de una vía férrea de $14 \mathrm{~km}$, que atravesaría pantanos, manglares, marismas y dunas entre las dos poblaciones (Poveda, 2002).

Con la capacidad técnica y la formación profesional de ingenieros alemanes traídos por la casa comercial, levantaron planos y mapas, hicieron el trazado de la línea, y estipularon especificaciones básicas de la carrilera. El 2 de febrero de 1869 se iniciaron los trabajos a orillas del caño de las Compañías en Barranquilla (donde se construye también la estación Montoya), y se terminan dos años después. Una obra que se hizo "a fuerza de músculos, con ayuda de picos, palas, sierras para cortar polines, lienzas, niveles manuales, niveles telescópicos de precisión, teodolitos ópticos y mucha disciplina alemana” (Poveda, 2002, p. 64).

El I de enero de I87I, se dio al servicio el ferrocarril desde Barranquilla hasta Sabanilla, luego rebautizada Salgar en honor del entonces presidente general Eustorgio Salgar. A lo largo de la vía se tendió la línea del telégrafo, otra innovación más que ayudaría a racionalizar el tiempo, ahora abstracto, para regular el despacho de los trenes y garantizar una circulación puntual y segura. 
El llamado Ferrocarril de Bolívar (en honor al Estado Soberano de Bolívar) desde sus inicios fue una operación muy exitosa: simplificó la operación portuaria, incrementó los volúmenes de importación y exportación, e hizo más eficiente las transacciones comerciales.

En la medida que aumentó la capacidad de carga de los barcos marinos, el calado de Sabanilla se hizo insuficiente; de modo que, en I884, se iniciaron otras obras para prolongar la carrilera 7 km más hacia la cercana bahía de Cupino, más amplia y estable, donde se construiría un muelle de $1220 \mathrm{~m}$, bodegas y terminales, que mejorarían considerablemente las instalaciones portuarias. En esta operación se vincularían el ingeniero cubano Francisco Javier Cisneros, como inversionista y promotor, y la compañía inglesa The Barranquilla Railway and Pier Company Limited, que contaban con el capital y la tecnología suficientes para emprender esa obra de ingeniería, innovadora para la época. La dirección técnica de la construcción del muelle estuvo a cargo del ingeniero inglés John B. Dougherty (Poveda, 2002).

Bautizado como "muelle de Puerto Colombia", se dio al servicio el I5 de junio de I893, contribuyendo durante más de cuatro décadas a impulsar el desarrollo urbano y la modernización de Barranquilla.

Es oportuno señalar que esa repentina importancia que, a fínales del s. XIX, Barranquilla adquiere como puerto y ciudad, no fue el resultado de un propósito de largo tiempo trazado por sus vernáculos habitantes, ni su tiempo evolucionó al ritmo del crecimiento vegetativo de su población. Este súbito protagonismo, en el comercio internacional al que fue arrojada, obedeció a las necesidades del tiempo del mundo, que la requería como un accidente, como un tránsito para el engrandecimiento de otros mundos. La Cámara de Comercio de Barranquilla la describía de esta manera:

Esta joven ciudad, llamada con acierto "hija de la República" erigida en Villa en 1813 y en ciudad en 1857, no tiene historia ni tradiciones coloniales. Surgió quizás por obra del acaso; su desarrollo data de 187 I merced a la inauguración del ferrocarril que la acercó al mar; había antes dormido durante dos siglos y medio el sueño de la ignorancia y el olvido; cobró alto vuelo en 1893 con la inauguración del gran Muelle de Puerto Colombia (Cámara de Comercio, 1938, p. 28).

Sin embargo, con esas condiciones de modernización temprana que le proporcionaron el muelle de Puerto Colombia y el Ferrocarril de Bolívar, la ciudad como proyecto urbano moderno comenzaba a ser viable y empezaría a cambiar su visión del mundo. Pues su noción de espacio y tiempo - del presente real, su significación y sentido-, no estaría tan ligada a su naturaleza física, ni a la cultura de sus pueblos ancestrales. Ahora comenzaba a tener un papel en el concierto internacional. Como Octavio Paz afirma,

para nosotros, hispanoamericanos, ese presente real no estaba en nuestros países: era el tiempo que vivían los otros, los ingleses, los franceses, los alemanes. El tiempo de Nueva York, París, Londres. Había que salir en su busca y traerlo a nuestras tierras (Giraldo \& Viviescas, 1995, p. II6).

De modo que, a partir de que se privilegió como puerto -a pesar de no tener condiciones naturales de bahía- y se incluyó en las rutas comerciales del mundo, Barranquilla tendría la imperiosa necesidad de hablar el mismo lenguaje universal comercial, utilizar el mismo léxico, emocionarse por las mismas cosas, sensibilizarse ante el mismo arte, configurar la 
misma arquitectura, utilizar la misma tecnología. La ciudad se montaría así en los afanes de la modernidad presentista, donde el pasado no cuenta, ni la naturaleza condiciona, aunque no dejaría de estar atrapada por los factores propios de un país subdesarrollado.

El imperativo del comercio internacional requirió que, en la ciudad y su territorio, se elaboraran cartografías más exactas, con la ayuda de instrumentos más sofisticados para medir el espacio, el ambiente natural. El sistema métrico decimal y las geometrías permitieron construir la geografía del lugar, apropiarse, manejar y modificar el territorio, domesticarlo y ponerlo al servicio de los intereses del capital.

Requerimientos implícitos en la noción de progreso que, desde el s. XVII, se había desarrollado como una teoría de la historia y una filosofía de la vida basada en la conquista de la racionalidad: "El progreso era el continuo desarrollo de la conquista de la naturaleza para ponerla al servicio del hombre, de la producción de bienes, de la producción de riqueza, de la producción de bienestar”, reflexiona O. Paz, (Giraldo \& Viviescas, I995, p. II5). Entonces, el ferrocarril y el muelle crearían esa geografía medida, abstraída, mapificada en el litoral occidental del departamento del Atlántico, para que Barranquilla se tornara en puerto y modernizara su estructura urbana.

Fue así como Barranquilla se convirtió en el principal puerto importador y exportador de Colombia (hasta finales de los años treinta), y en un polo de atracción para los habitantes de la región Caribe, para los extranjeros aventureros, y para las gentes del interior del país. En 1903 se exportaba el $60 \%$ de las cargas de café, gracias a políticas públicas de la nación que promocionaban este tipo de comercio; y ciertas políticas proteccionistas favorecieron la instalación de industrias (Villalón, 2000).

\section{Los tajamares y la unión del puerto marítimo y fluvial}

\section{La obsesión de Barranquilla es convertir su acceso al mar en propósito nacional (Segovia, 1999, p. 3).}

Si bien era cierto que el ferrocarril y el muelle habían mejorado la eficiencia portuaria y facilitado la conexión de dos puertos -el marítimo en Puerto Colombia, y el fluvial en Barranquilla-, se trataba ahora, sin embargo, de unir de manera fluida el tráfico marítimo internacional (el mercado mundial) con el tráfico fluvial (el mercado nacional) en el río Magdalena.

Esta conexión fluida entre mar y río era el referente de la modernización en todas partes del mundo, que se incorporaba de manera dinámica al comercio internacional, mejorando las condiciones de navegabilidad y barimetría en la desembocadura de los ríos. Ejemplos se hallan en Nueva Orleans, en el río Misisipi con el golfo de México; en Róterdam, el río Mosa con el canal de la Mancha; en Nueva York, el río Hudson con el océano Atlántico; en Hamburgo, el río Elba con el mar del Norte; en Boston, el río Charles con la bahía de Massachusetts; en Londres, el río Támesis con el mar del Norte.

El río Magdalena había sido desde tiempos precolombinos, y durante la Colonia, la principal vía de comunicación fluvial para las poblaciones de las sabanas del Caribe colombiano y de la parte baja de los Andes Occidentales; más aún, en la temprana república sería pensado como la columna vertebral del desarrollo económico de la Costa Norte. Teniendo en cuenta que el bajo Magdalena está irrigado por una red importante de ríos, afluentes 
y ciénagas, que, interconectadas, posibilitarían la navegación a vapor y el transporte de carga y mercancías pesadas.

Pero ante la necesidad de vincular más la economía nacional con los mercados internacionales, a finales del s. XIX, el río ya no sería pensado solo como eje del mercado interno regional, sino también como la arteria que podía comunicar con el mundo exterior. Ahora se consideraba fundamental para el proyecto de modernización colombiana. Tan importante era conectar, a través del río, el mayor número de poblados colombianos en el interior del país', como alcanzar el mar: paisaje y horizonte del que provenían los bienes de capital, las mercancías, las ideas y los objetos de mundo moderno, deseados por la novísima república liberal colombiana.

Conectar el mar con el río se convertiría entonces en un ideario político prioritario. Era fundamental para mejorar la integración de la economía nacional a las redes comerciales mundiales; lo cual, si bien ya se estaba haciendo a través de Cartagena, Puerto Colombia y Santa Marta, no lo era de la forma más eficiente. Significaría también ampliar el horizonte pensante del estrecho mundo colonial, al mundo desarrollado de Europa y Norteamérica. Se trataba ahora de conectar lo universal con lo nacional, la oferta con la demanda, trocar el café por maquinarias.

Entre 1877 y 1886 , en la desembocadura del río se presentó, de manera natural, una remoción de las barras de arena, que permitió el acceso de 459 buques hacia Barranquilla. Esta situación generó entusiasmo entre los comerciantes locales y avivó las esperanzas de un tráfico fluido de embarcaciones. Pero, en I887, la reaparición de la barra de arena hizo nuevamente imposible que los buques de gran calado entraran al Magdalena. Esta apertura ocasional, sin embargo, estimuló "el interés público por la apertura de Bocas de Ceniza como sustituto del ferrocarril y del muelle de Puerto Colombia" (Posada, 1998, p. 284).

Motivados por esa posibilidad, en 1906, los dirigentes de Barranquilla financiaron unos primeros estudios hidráulicos, contratando a los ingenieros norteamericanos Ripley y Haupt. Luego, en 1915, la firma alemana de Julius Berger los complementó. Después de la Primera Guerra Mundial, los comerciantes locales registraron la Sociedad Colombiana de Bocas de Ceniza (1919) y contrataron con el Gobierno colombiano la adecuación de la desembocadura. El punto de partida serían los estudios de Berger. Pero, ya en 1916, en el II Congreso Científico Panamericano, el ingeniero colombiano Miguel Triana había sugerido reparos a dichos estudios. Ahora -anota R. Segovia-, los banqueros de la obra exigen revisiones, $y$, para ello, encargan de los diseños a Black, McKenney and Stewart -veteranos del US Army Corps of Engineers-, quienes optaron por un canal de 880 metros, encauzado por dos tajamares rectos de aproximadamente 2300 m cada uno (1999).

En 1925, el Gobierno nacional adjudicó el contrato de las obras civiles a Ulen \& Co. No obstante, en 1929, por los efectos de la crisis mundial, el incumplimiento del contratista y el incremento en los costos ocasionaron que el Ministerio de Obras diera por terminado el contrato de la Ule, y las obras de los tajamares se paralizaron.

Esta parálisis fue motivo de agrias disputas entre los dirigentes locales y el Gobierno nacional, pues los primeros acusaban al alto Gobierno de entorpecer y recortar el financiamiento de las obras. Consideraban que las negociaciones y los trámites eran dilatados. Por su lado, el diario La Prensa denunciaba que el tema de Bocas de Ceniza se había convertido

I A finales del s. XIX no existía una red de carreteras para vehículos motorizados que uniera las distintas poblaciones de Colombia. 
"en la más insoluble de las incógnitas y en el más complicado negocio de la República", transcribe E. Posada (1998, p. 289). Y añade el mismo autor que los congresistas costeños se quejaban de que había mayores partidas presupuestales para financiar las líneas férreas y las primeras carreteras en el interior del país. Como consecuencia, el 22 de octubre de 193I, en Barranquilla hubo una gran manifestación cívica -que desembocó en violentos disturbios-, en la que participaron todos los estamentos sociales, y, además, contó con la simpatía del gobernador del Atlántico.

Barranquilla, entonces, recurrió al presidente liberal Enrique Olaya Herrera; $y$, en 1933 se pudo firmar un contrato con el empresario norteamericano, residente en la ciudad, Robert Parrish, en representación de la firma Raymond Concrete Pyle y Winston Brothers, para la apertura de Bocas de Ceniza y la construcción de instalaciones portuarias adicionales -muelles, bodegas, dársenas-. En diciembre de 1936 los tajamares de Bocas de Cenizas se inauguraron oficialmente.

La construcción de ese canal navegable en la desembocadura del río significó un impulso mayor para continuar la modernización de la economía, de la construcción, y del comercio de la ciudad, a partir de la década de los treinta ${ }^{2}$. Fue una obra de privilegio para Barranquilla, pues la mejora ostensible de su infraestructura portuaria posibilitó, aún más, su interacción comercial con el mundo occidental. La importación de maquinarias y tecnologías industrializadas para el sector de la construcción, de materiales y bienes de consumo, de electrodomésticos, vehículos, alimentos enlatados, entre otros, se daba ahora con más facilidad, lo cual la hacía más próxima a la modernización que otras ciudades de la región y del país ${ }^{3}$.

Con esta inversión, Barranquilla se sentía preparada para ser el principal puerto exportador e importador de Colombia, lo que le daría una posición de liderazgo dentro del engranaje de la economía nacional. Sin embargo, desde 1935, habría de perder su primacía portuaria a manos de la triada: canal de Panamá, Ferrocarril del Pacífico y Puerto de Buenaventura, por donde saldría ahora el mayor volumen de sacos de café -principal fuente de ingresos de la economía nacional en el s. $X X^{4}-$. Fue esta una de las principales razones por las cuales se reorientó la inversión pública nacional en obras de infraestructura vial hacia el Pacífico, lo cual desestimuló el tráfico por el río y los puertos del Caribe. Señala Eduardo Posada Carbó:

A medida que en el interior se desarrollaban nuevos medios de transporte, la región iba perdiendo sus ventajas: las exportaciones de café abandonaron gradualmente los puertos del Caribe y los productos regionales debieron enfrentar costos crecientes en el transporte para llegar a los Andes (Posada, 1998, p. 290).

Para contrarrestar esta tendencia centralista, Tomás Surí Salcedo, dirigente local de la época, proponía que, además de la apertura de Bocas de Ceniza, se adelantaran trabajos de regularización del río Magdalena. Pues era la principal fuente de comunicación de una serie de poblados en una región que se identificaba por su carácter anfibio ${ }^{5}$ y con los cua-

2 Significará también la muerte comercial del muelle de Puerto Colombia y de su línea férrea de conexión con la ciudad: símbolos de la primera modernidad del s. XIX.

3 Sumado al hecho de que Barranquilla era el principal puerto aéreo internacional de Colombia hasta bien entrada la década de los sesenta, como se verá más adelante

4 El puerto de Barranquilla comenzó a perder primacía para la economía colombiana cuando el canal de Panamá se dio al servicio en 1914. Al año siguiente, se construiría el Ferrocarril del Pacífico para conectar las zonas cafeteras con el puerto de Buenaventura, y a partir de 1935 este lo superaría en los volúmenes de carga de exportación e importación.

5 El bajo Magdalena tiene la función ecológica de servir de zona de desborde natural de los ríos Magdalena, Cauca, San Jorge, Sinú, Cesar, y sus afluentes. El desbordamiento en invierno fertiliza las Ilanuras y renueva el agua de las ciénagas. 
les -pensaba Salcedo- se podía avivar el comercio y el desarrollo del hinterland mediante la navegación a vapor por el río.

Karl Parrish consideraba que "la parte baja del valle del río Magdalena y sus tributarios es la mayor zona de tierras ricas para la agricultura con posibilidades de mecanización en Colombia"; por lo tanto, Bocas de Ceniza "abriría este vasto territorio al transporte marítimo. Esto proporcionaría una salida al mar para los productos que llegan desde Magangué, el Saint Louis de Colombia..." (Posada, 1998, p. 292).

La realidad fue que, a finales de los años cincuenta, Barranquilla quedó incrustada como una isla de modernidad -con sus propios bordes premodernos, en donde los esfuerzos por vincular de manera eficaz los recursos y los potenciales regionales no fueron suficientes-. Permanentes fueron los problemas de calado de Bocas de Ceniza que hacían ineficiente el puerto; el río no fue posible regularizarlo y las inversiones en redes ferroviarias fueron exiguas, comparadas con las de la región Andina. Las carreteras comenzaron a ser aceptables solo en la segunda mitad del s. XX.

Desvinculada de la dinámica económica del país, su aislamiento fue motivo de preocupación del ingeniero antioqueño y expresidente de la Federación Nacional de Cafeteros, Alejandro González, quien consideraba ese aislamiento un problema de soberanía nacional, pues la Costa Caribe (Barranquilla incluida) quedó "a merced de las influencias exteriores" (González en Posada, 1998, p. 29I). Esa circunstancia podría mirarse desde otra óptica: tal vez fue mejor para los propósitos de fortalecer una identidad local y aceptar como positivas esas influencias exteriores.

\section{La aviación: el atajo al aislamiento del delta}

Uniremos muy pronto con las ondulantes cintas de nuestro hermoso Magdalena, las ardientes playas del mar Caribe y las risueñas montañas de Antioquia y Cundinamarca. Llevaremos a nuestros hermanos del interior el saludo matutino del sol naciente para recoger allá los últimos rayos del astro Rey, cuando en los campanarios floten los dulces toques del Ángelus (Cortissoz, 1919 en Agudelo, 2010, p. 2).

La zona del bajo Magdalena, que conforman los departamentos de Bolívar, Atlántico y Magdalena, constituye un área de desborde natural de los ríos Magdalena, Cauca y San Jorge, que en épocas de invierno inundan las llanuras y conforman una especie de mar mediterráneo interno. Este -antes de la mecanización de los transportes- obligaba a las poblaciones asentadas en el valle a movilizarse en canoas, chalupas, balsas, champanes, o bongos, pues la comunicación terrestre se tornaba casi imposible por lo precarios que eran los caminos y las trochas ${ }^{6}$.

La economía moderna del siglo XX no podía detenerse ante los ciclos de la naturaleza. En la más poblada Colombia andina y el mundo occidental, el comercio era un imperativo para la república y la región. Sin unas redes de comunicaciones eficientes, veloces y mecanizadas que integraran las dispersas comarcas colombianas, el proyecto de Estado-nación abortaría. Se pensaba que el concepto de nación solo podría materializarse en Colombia si era mediado por la mecanización del transporte, ya que el incremento de la velocidad

6 El sociólogo barranquillero Orlando Fals Borda señalaba cómo esa doble condición del medioambiente del Caribe colombiano determinó la formación de una cultura anfibia, en su libro La historia doble de la Costa (2002). 
acercaba a las comunidades, transformaba el paisaje y acotaba la experiencia del territorio. De hecho, así era visto por la revista de arquitectura Proa, que consideraba: "La nación solo existía al interior de unos límites construidos por la modernidad, y por lo tanto, no había nación posible más allá de los límites de la modernidad", como anotan J. Arango y C. Martínez, citados por Mondragón (2007, p. 6).

Marchando entonces por esa senda de la modernización, a finales del s. XIX, la navegación por el río Magdalena había mejorado gracias a la introducción de vapores y planchones que dinamizaron el comercio de la región con el interior del país, no obstante, la inestabilidad del calado del río. Desde I893, el ferrocarril había permitido una comunicación eficiente entre el puerto marítimo de Sabanilla y el puerto fluvial de Barranquilla. Pero las comunicaciones terrestres de los centros más poblados del Caribe entre sí, y de estos con el interior del país, seguían siendo las mismas desde la Colonia.

Con la llegada de la aviación a Colombia, en la segunda década del s. XX, se produjo un significativo salto adelante en la construcción de ese sueño de nación moderna. Porque ese hecho permitió tomar conciencia de un territorio unificado, ya que por el aire se podían superar los macizos montañosos colombianos, y romper el aislamiento físico que las ciénagas, lagunas o humedales le generaban a la región. Por el aire se llegaría más rápido y más lejos, al resto del mundo. De modo que, como anotan Arango y Martínez: "El país se hizo plano y las distancias, que antes se contaban por semanas o meses, comenzaron a contarse por horas o por fracción de hora. Lo que era apartado, vasto, inalcanzable, se hizo familiar" (Mondragón, 2007, p. 5).

Por su posición geográfica, por el dinamismo de su economía y por su cosmopolitismo, Barranquilla se convertiría en protagonista principal de ese proceso de modernización: el 5 de diciembre de 1919 tres alemanes y cinco barranquilleros registraron en la Notaría Segunda de la ciudad, la Sociedad Colombo Alemana de Transporte Aéreo, Scadta ${ }^{7}$. Esta sería la tercera aerolínea del mundo, después de la SPT Airboat Line (EE. UU.) y KLM (Holanda), y la primera compañía aérea de Suramérica.

Scadta comenzó como una pequeña empresa transportista aérea con hidroaviones Junkers F-I 3 de fabricación alemana -los primeros aviones metálicos del mundo-, habilitados para aterrizar en ríos y cuerpos de agua. Estos primeros aeroplanos, fabricados en duraluminio, tenían capacidad para cuatro pasajeros, dos tripulantes y una tonelada de carga; además de la extraordinaria comodidad de navegar bajo techo, a resguardo de las inclemencias del tiempo (Scadta.co, 20I2).

Después de pequeños vuelos de prueba, incluyendo uno entre Puerto Colombia y Barranquilla, el primer vuelo oficial de Scadta se llevó a cabo el 9 de septiembre de 1920, entre Barranquilla y Puerto Berrío, siguiendo el curso del río Magdalena. Un viaje que duró dos días, ya que hizo escala en Magangué, Zambrano, y pernoctó en El Banco antes de llegar a Puerto Berrío, haciendo una parada en La Gloria. El tiempo acumulado de vuelo fue de seis horas. Llevaba dos tripulantes y un pasajero, y el primer encargo de correos aéreos (Scadta.co, 20I2).

7 Los señores Werner Kammerer, Albert Tietjen, Stuart Hosie, Ernesto Cortissoz, Jacobo Correa, Aristides Noguera y Rafael Palacio constituyeron la Sociedad Colombo Alemana de Transportes Aéreos. La tripulación de los aviones la constituían experimentados pilotos alemanes que habían participado en la Primera Guerra Mundial, lo mismo que los mecánicos e ingenieros que le daban soporte y mantenimiento, como lo registra Mauricio Sáenz en Semana.com, consultada en dic., 2012 . 
El 20 de octubre de ese mismo año, se inició una ruta semanal que partía de Barranquilla, y acuatizaba diez horas más tarde en Girardot; de donde se continuaba en tren, otras diez horas, hasta llegar a Bogotá. Un viaje que, comparado con las dos semanas que demoraba un vapor por el río Magdalena para el mismo trayecto, significaba un notable ahorro de tiempo. Por esta razón, se comenzó a generar una demanda para este servicio. El I 2 de noviembre se amplió la ruta Girardot-Bogotá, haciendo el cambio de flotadores a ruedas en la aeronave.

Diez años más tarde, Scadta ya había realizado 2266 viajes comerciales, transportado 5102 pasajeros, 59484 kilos de correos, y ampliando la oferta a otros destinos nacionales, como Ibagué, Buenaventura, Cartagena y Neiva, e internacionales, como Balboa y Cristóbal en la zona del canal de Panamá ${ }^{8}$.

Como el río Magdalena constituía una pista de aterrizaje natural, el primer hidropuerto de Barranquilla se estableció, en I92I, en el sitio denominado "Veranillo", en la orilla occidental del caño de las Compañías. Su infraestructura básica consistía en una serie de hangares para proporcionarles mantenimiento a los hidroplanos; $y$ bodegas con cubiertas a dos aguas para las actividades complementarias de almacenamiento y servicios. Un conjunto de palmeras, a la entrada de las edificaciones, le imprimía una nota de paisajismo tropical. Luego, en 1930, se construyó un hangar de mayores dimensiones, una estructura metálica importada de Estados Unidos, que sustituyó los galpones iniciales a orillas del caño (Scadta.co).

Barranquilla entonces se convirtió, desde 1920 hasta 1935, en el puerto multimodal por excelencia de Colombia. El hidropuerto de Veranillo se encontraba a escasos $2000 \mathrm{~m}$ de la Intendencia Fluvial, por donde se hacían las conexiones con el resto de poblaciones ribereñas, y a través de estas con las principales ciudades andinas colombinas; quedaba asimismo a 1700 metros de la Estación Montoya, en la que se hacía la conexión con el terminal marítimo del muelle de Puerto Colombia, adonde llegaban extranjeros y mercancías del resto del mundo. La ciudad estaba integrada con los tiempos de la modernización y le abría camino al resto de Colombia.

No obstante, este nodo intermodal (avión, tren, barco) comenzaría a romperse muy pronto, en 1936, cuando los tajamares de Bocas de Ceniza tornaron inútil el Ferrocarril de Bolívar, al permitir la llegada de barcos marítimos directo al río Magdalena. Con la construcción, en 1945, de la pista de aterrizaje y el aeropuerto "Las Nieves", en el municipio de Soledad, y gracias a que por el avance tecnológico de la aviación ya no se requería de las superficies lisas de los cuerpos de agua para aterrizar, se volvió así, muy pronto, obsoleto el hidropuerto de Veranillo.

Después de una historia de intrigas, presiones políticas, disputas accionarias, y tras el ataque japonés a Pearl Harbor, Scadta se vio obligada a cesar sus operaciones; y sus activos fueron combinados por el Gobierno colombiano en la aerolínea estatal SACO, formando la moderna línea aérea nacional de Colombia: Avianca.

La aviación fue un fenómeno tecnológico y social que jugó un papel determinante en la modernización, trastocando las lógicas urbanas de la ciudad y los paradigmas de la arquitectura moderna. El movimiento italiano del futurismo, ya lo había reconocido en el Manifiesto futurista de la arquitectura aérea del I de febrero de 1934, a saber:

8 Datos del Informe de Scadta de 1930 a la Asamblea General de Accionista (Matacafé.co, 2012). 
[...] hoy se añade un factor importante: la Aviación. Esta modifica el mundo civil y militarmente, plantea nuevos problemas artísticos, sociales, políticos, industriales y comerciales. Los poetas, arquitectos y periodistas futuristas hemos creado la gran Ciudad única de líneas continuas para ser contemplada en el vuelo, fuga paralela de Aeropistas y Aerocanales de cincuenta metros de ancho, separados entre sí por ágiles urbanizaciones de repostaje (repostaje espiritual y material). [...] Por razones estéticas y para armonizar cada vez más empáticamente la vida de la tierra con la vida del cielo, los puertos marítimos y de hidroaviones ya no tendrán inmóviles acantilados, sino móviles muelles de acero que ofrecerán abrigo a los hidroaviones desde cualquier dirección [...] (Marinetti, F.T. \& Mazzoni, A, 1934).

En Barranquilla, la aviación fue fundamental para cerrar el círculo virtuoso de los transportes mecanizados, con los cuales transformar y adecuar su territorio a las exigencias del capitalismo mundial: tiempos rápidos, medios eficaces e información oportuna; de modo que se le facilitó, antes que a otras ciudades colombianas, la llegada de la cultura de la racionalidad moderna, los extranjeros pioneros de su desarrollo, las mercancías, la tecnología, y los deseos de vivir en un hábitat moderno, tal como sería la intención de la arquitectura que se construiría en el s. XX.

\section{CONCLUSIONES}

Los deltas de los ríos han jugado un papel fundamental en la consolidación de las civilizaciones, pues estas se enriquecen con el intercambio de bienes materiales de culturas distantes -separadas por mares y océanos- que se propician en sus puertos, permitiendo que en el interior de los territorios que recorren los ríos circulen las mercancías, los mercaderes y sus culturas. Barranquilla confirma esa constante histórica y se evidencia en el hecho de que el país nacional moderno que se empezó a delinear en el siglo $X X$, tuvo su principal acceso a la tecnología, los bienes de capital y artefactos industrializados a través de su puerto marítimo, fluvial y aéreo.

La Barranquilla de hoy debe su razón de ser al papel que jugó su localización como puerto del delta del Magdalena, a finales del siglo XIX y principios del $X X$ a pesar de que ya en el siglo XXI, el avance de las tecnologías de transporte y comunicación hayan borrado las ventajas comparativas de su geografía. El Internet rompió el aislamiento cultural de las ciudades continentales, el centralismo del distrito capital de Bogotá le arrebató su primacía aeroportuaria, las carreteras desplazaron al río Magdalena como principal ruta de transportes y los otros puertos de Colombia se tornaron más competitivos por la dificultad constante de mantener un calado adecuado en Bocas de Ceniza, amén de mayor tamaño de los barcos actuales.

Barranquilla bajó de rango en su importancia comercial y portuaria para el país, pero logró consolidarse como un conglomerado urbano con una cultura muy particular, cosmopolita, mestiza y caribeña, con nuevos desafíos que afrontar en un mundo más globalizado y competitivo. 


\section{REFERENCIAS}

Aprile, J. (1992). La ciudad colombiana. Siglo XIX y XX. Bogotá: Biblioteca Banco Popular.

Arango, J., \& Martínez, C. (1949). Arquitectura en Colombia. Recuperado de: https://groups. google.com/forum/\#!msg/soc.culture.colombia/

Cortissoz, E. En Agudelo, L. (2010). Artículo histórico sobre Avianca. Recuperado de http:// newsgroups.derkeiler.com/Archive/Soc/soc.culture.colombia/20 I I-0

Fals Borda, O. (2002). La historia doble de la Costa. Bogotá: Áncora Editores, Universidad Nacional.

Giraldo, F., \& Viviescas, F. (1995). La modernidad en Colombia. Bogotá: Foro Nacional Por Colombia.

Marinetti, F. T., \& Mazzoni, A. (febrero, 1934). Manifiesto Futurista de la arquitectura aérea. Sant'Elia, II(3).

Mondragón, H. (2008). Arquitectura en Colombia I946-195 I: Lecturas críticas de la revista Proa. Revista de Arquitectura, 2). Universidad de los Andes.

Oeding, K. M. (s.f.). Barranquilla cuna de la aviación civil. La historia de Scadta. Recuperado el I0/I2/I2 de: http://www.barranquilla-online.com/BOL/

Paz, O. (199I). La búsqueda del presente. En F. Giraldo, F. Viviescas (comp). Colombia al Despertar de la Modernidad. Foro Nacional por Colombia. Bogotá: Carvajal.

Posada Carbó, E. (1998). En el Caribe Colombiano. Diario La Prensa. (Septiembre 9 de 1930).

Poveda, G. (noviembre, 2002). El primer ferrocarril en Colombia. Dyna, 69(I37), 6I-73. Universidad Nacional de Colombia. Medellín.

Romero, J. (1999). Ciudades e ideas en América Latina. Medellín: Universidad de Antioquia.

Scadta. (s.f.) Datos del Informe de Scadta de 1930 a la Asamblea General de Accionista. Recuperado el I0/I2/I2 de www.matacafé.co

Segovia, R. (1999). Los tajamares de Bocas de Ceniza. Revista Credencial, 35, 3.

Villalón, J. (1992). Historia de Barranquilla. Barranquilla: Ediciones Uninorte. 